\title{
Analysis of Intensity and Vibration of 190T Electric Wheel Dump Truck Frame
}

\author{
X. G. $Y u^{1, a}$, L. Yang ${ }^{1, b}$ \\ ${ }^{1}$ School of Mechanical Engineering and Automation, University of Science and Technology \\ Liaoning, Anshan, Liaoning, 114051, China \\ a yuxiaoguang58@163.com, bhbyanglei@163.com
}

Keywords: Frame, Crack, Finite element, Modal analysis, Random vibration

\begin{abstract}
Through the cracking failure analysis of dump truck frame with large electric wheel, the static and dynamic frame analysis of performance evaluation are conducted by the finite element analysis method. From the strength and stiffness analysis frame structure in the weak links, it is easy to find out crack the parts and the reasons with frame, and through the modal analysis and random vibration and fatigue strength analysis the danger frequency of the frame induced by road encourage is obtained, which can prevent the occurrence of frame cracks and provide a foundation for further optimization of the frame.
\end{abstract}

\section{Introduction}

$190 \mathrm{~T}$ electric wheels dump truck is the main transport equipments in mines. As the carrying capacity, the car must not only withstand high shock and vibration excitation, coupled with the alpine zone is located in the northeast, difficult operating environment, requires the car frame with high fatigue strength and low temperature impact toughness. For the prevention and early detection of cracks, need to analyze the reasons for cracking[1].

\section{Finite element modeling}

Finite element method is used to frame three working condition analysis, bridge and suspension devices all are simulated using beam elements, in connection with the frame by hinge coupled with the simulation. Finally, face the constraints of the frame and wheels connected to the transfer to the axle, the dynamic load factor based on road conditions, and generally 1.2 to 3.5 , mesh and load frame as shown in Fig.1 [2, 3].

\section{Finite element analysis results}

(1) The pure bending working condition is refers to the four wheels of the vehicle on the same plane of static condition; Bending to the constraint conditions for: constraints the right front supporting points in the integral coordinate system $\mathrm{X}, \mathrm{Y}$ and $\mathrm{Z}$ freedom, constraint the right rear supporting points $\mathrm{Y}$ and $\mathrm{Z}$ freedom, constraint the left front supporting points $\mathrm{X}$ and $\mathrm{Z}$ freedom, and constraint after left supporting points $Z$ freedom. (The use analysis of coordinate system: the axis of the $X$ is the advance orientation; the $\mathrm{Y}$ axis of the vertical paper is in the face of positive direction, $\mathrm{Z}$ axis of the up vertical direction). The analysis of the strain and equivalent stress has shown in Fig.2 and Fig.3. 


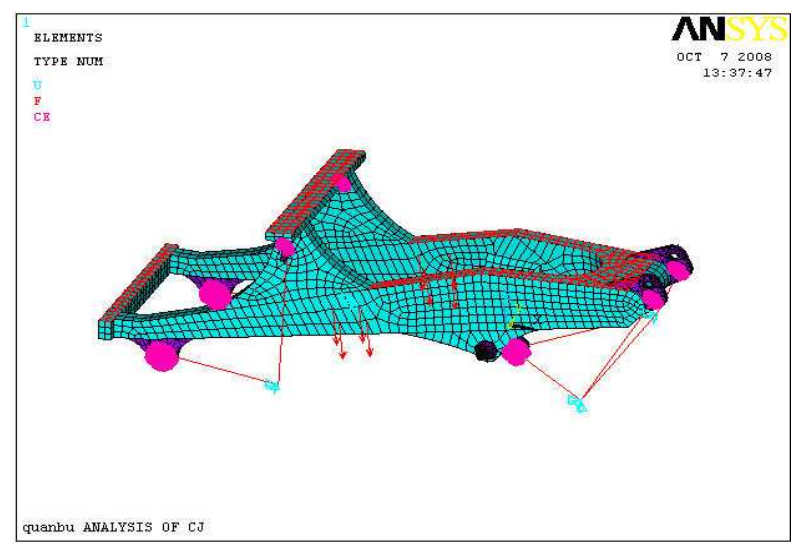

Fig. 1. Frame of the grid division and Load map

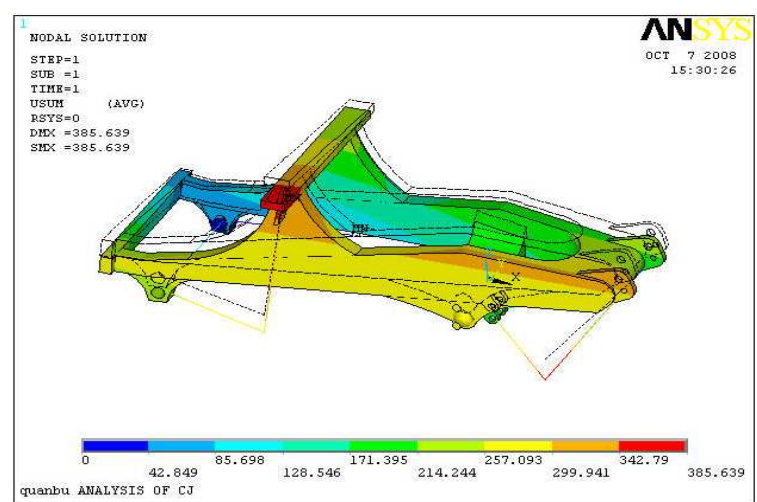

Fig . 2. The frame strain with bending

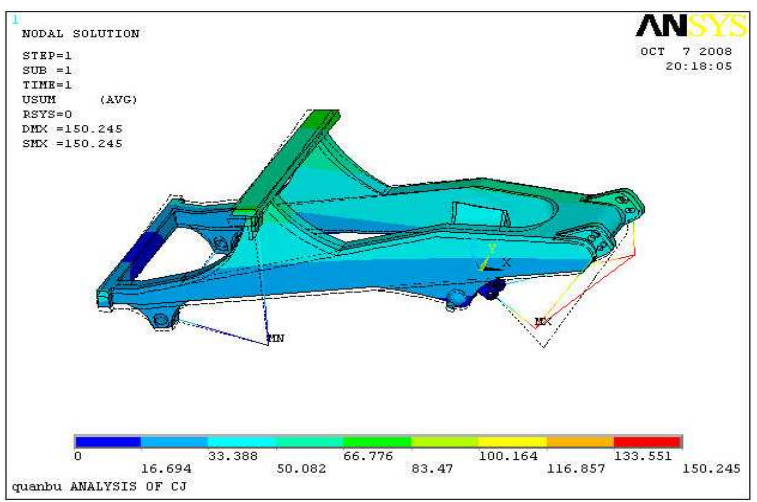

Fig. 4. The frame strain with reverse

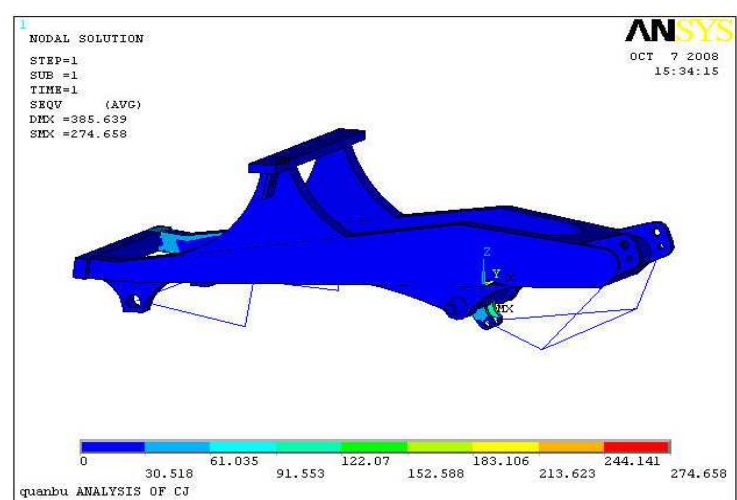

Fig. 3. The frame equivalent stress with bending

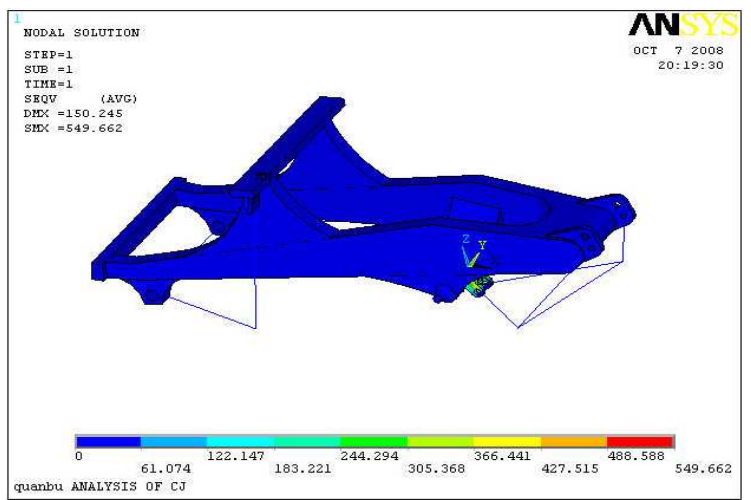

Fig. 5. The frame equivalent stress with reverse

The main of the goods loading is load on the back of the frame, and other load relatively small and cause deformation after from before, is becoming to smaller. By bending condition influence, after the direction tilt left frame, the left bridge piece of frame is the biggest deformation, deformation of $98.3 \mathrm{~mm}$, and overall maximum deformation $385.6 \mathrm{~mm}$. It is largely due to the smaller oil and gas suspension stiffness and the deformation of axle institutions, the frame deformation is smaller The maximum stress of the frame is $274.6 \mathrm{MPa}$, less than $680 \mathrm{MPa}$ yield limit It is explain the frame is safe under the load conditions The frame and rear axle shaft to lift part of the biggest, but the connected rear bridge, type A frame and lifting suspension can reduce load through to absorb energy and cause the large deformation. Although by bending condition obviously, but frame have overall strength evenly, can through the axle and suspension system to cushion the impact of incentive. 
(2) The boundary conditions of the reverse working conditions for two front stationary, and let the rear side up the $150 \mathrm{~mm}$, another side fell $150 \mathrm{~mm}$, other constraints, the analytical results released as shown in Fig. 4 and Fig.5.

The maximum variables is $150.2 \mathrm{~mm}$ to the condition, mainly for the rear bridge deformation, the frame deformation is $83.47 \mathrm{~mm}$ The greatest stress for $549.6 \mathrm{MPa}$, still not exceeding the admissible stress also meet stress strength requirement, because of the constraint to wheel strong point, lead to the frame have a larger deformation on the back end and the top, but the frame in addition to lifting roller and lifting lugs shaft block appear local stress the larger, other strength evenly, explain the frame stability is well, the stress can through the rear axle buffer in order to reduce the impact of deformation. 3) the right rear wheel hang in the air working condition release all restrain to the right rear wheel, impending all constraints to other wheel, observation constraint force of the state in impending, as shown in Fig.6 and Fig.7.

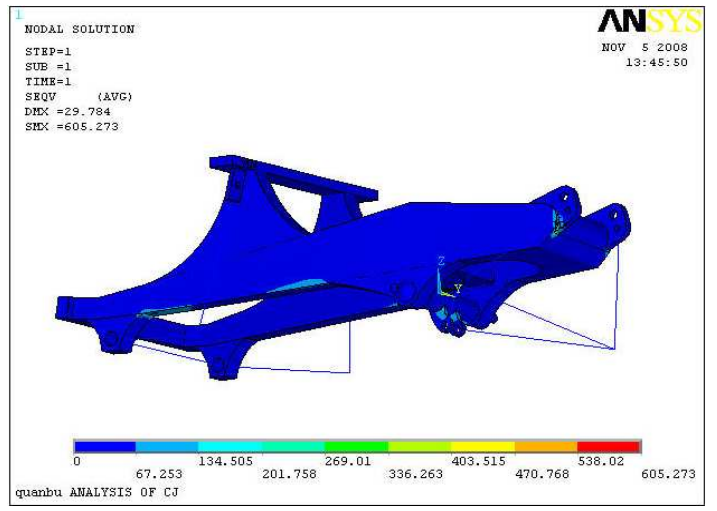

Fig. 6. The frame equivalent stress with right rear suspension

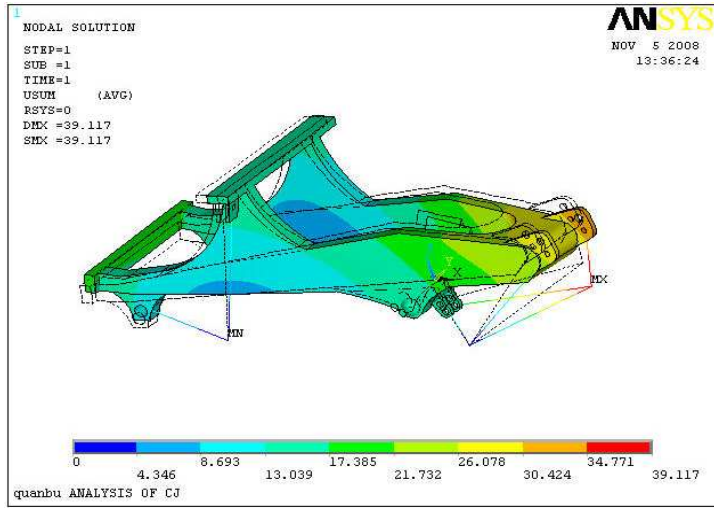

Fig. 7. The frame strain with right rear suspension

Through the above two figure shows that the frame deformation is smaller relative to other two conditions under the impending, mainly because the overall rear axle coherent function have very good stable effect, but the equivalent stress is bigger, the maximum for $605.273 \mathrm{Mpa}$, appear in the border of the left big tip seat bottom and frame; one end suspended, the deformation is more apparent, but the opposite angles is not big that explai frame structure frame of distortion resistance ability is good, but stress mainly passed to the other side led to another side force, easy to cause too much stress. Due to the dump radius and wheel pressure big, to temporarily suspended can through the other one end of the tire support, as soon as possible reduce the impact of impending working conditions.

Comprehensive analysis, in the above dangerous conditions, the frame stress will meet strength and rigidity requirements, above all working conditions is the frame overloaded performance of stress and strain situation, we can see that the impending condition is the most dangerous working conditions and improvement the dump road. The actual working condition, the above conditions may stack or alternate repeat and more influence to frame. so it is necessary to frame in driving force on the experiment test, to clear frame stress state. Of the three conditions are not directly destroy frame, so that static stress is not enough to rupture, and the occurrence of crack should be the dumper produces dynamic alternating stress in the process of run. 


\section{The frame modal analysis}

The dumper frame of electric wheels is elastic vibration system of more degree of freedom, apply to the various system vibration force is the frame to produce complex vibration of the power supply. Cause all kinds of exciting force can be summarized as factors of two types: one is When the dumper is driving, the road to the role of the unevenness of the rando mvibration; 2 when is the engine running, the harmonic excitation is caused of working stroke burning breakout pressure and the piston reciprocating inertia force. If these vibration frequency with exciting force and a natural frequency of the frame is coincide from resonance, and led to a the frame is destroyed Therefore, the frame dynamic design requirements the natural frequency And vibration model of the frame should avoid common frequency of excitation force, so that we can guarantee the frame has good dynamic characteristics[4-6].

This paper established the finite element modal analysis model with $190 \mathrm{~T}$ dumper frame, using ANSYS finite element calculation software get the natural frequency and vibration mode of frame , and make assess to the dynamic characteristics of the frame.

Table 1 Frequency values of frame in the initial 15 orders

\begin{tabular}{ccccc}
\hline 1st Order & 2nd Order & 3rd Order & 4thOrder & 5th Order \\
\hline 0 & $0.14863 \mathrm{E}-03$ & $0.17715 \mathrm{E}-03$ & $0.33114 \mathrm{E}-01$ & $0.34916 \mathrm{E}-01$ \\
6th Order & 7 th Order & 8th Order & 9th Order & 10 thOrder \\
0.10390 & 22.906 & 29.041 & 35.698 & 37.827 \\
11 st Order & 12 nd Order & 13 rd Order & 14 th Order & 15 th Order \\
44.306 & 50.932 & 54.269 & 60.207 & 61.848 \\
\hline
\end{tabular}

190 T frame modal analysis mainly includes four steps: building a model, loading solution, and expansion mode and observation results. The established model is previously established entity mode of $190 \mathrm{~T}$ frame as the modal of the model analysis, the meshing finite element model include 11234 units, 23811 node. The loading solving modal analysis displacement constraints temporary not impose, by eliminating the rigid body vibration model of the corresponding frequency value to get flame natural frequency and vibration mode, make the method of Lanczos the modal analysis, ignore damping effect extract before 15 orders nature frequency and vibration mode. Observe the results calculated by ANSYS get frame 15 orders before modal frequency as shown in Table 1.

\section{Experiment test}

The experiment is testing a stress distribution condition of the frame easy to crack part, which is make stress distribution electrical testing to frame, the arrangement of the strain gauge is according to the early fracture actual ocations in the process of working, and the large stress station in finite element analysis. 

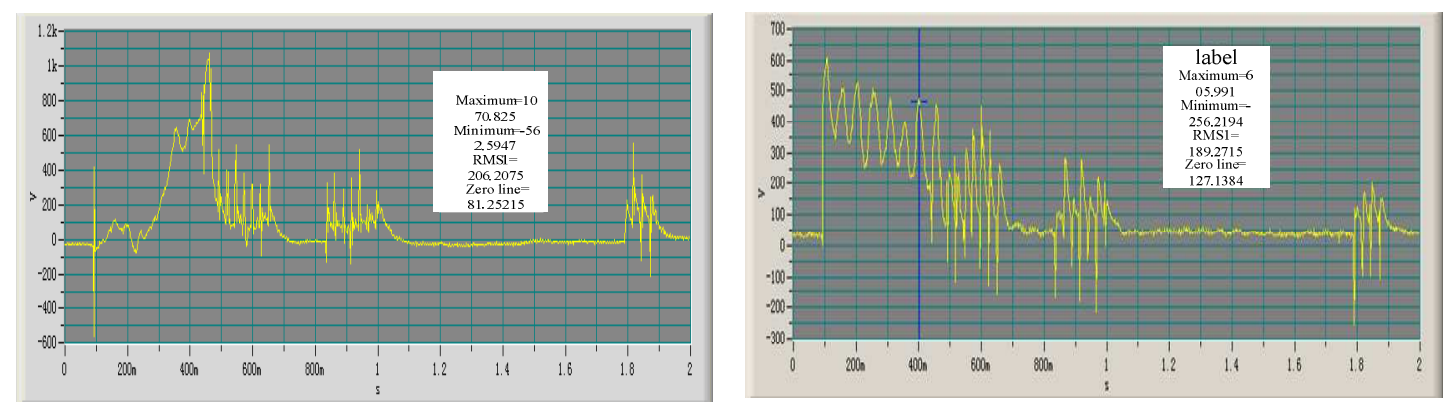

Fig. 8. Measure data curve with noload
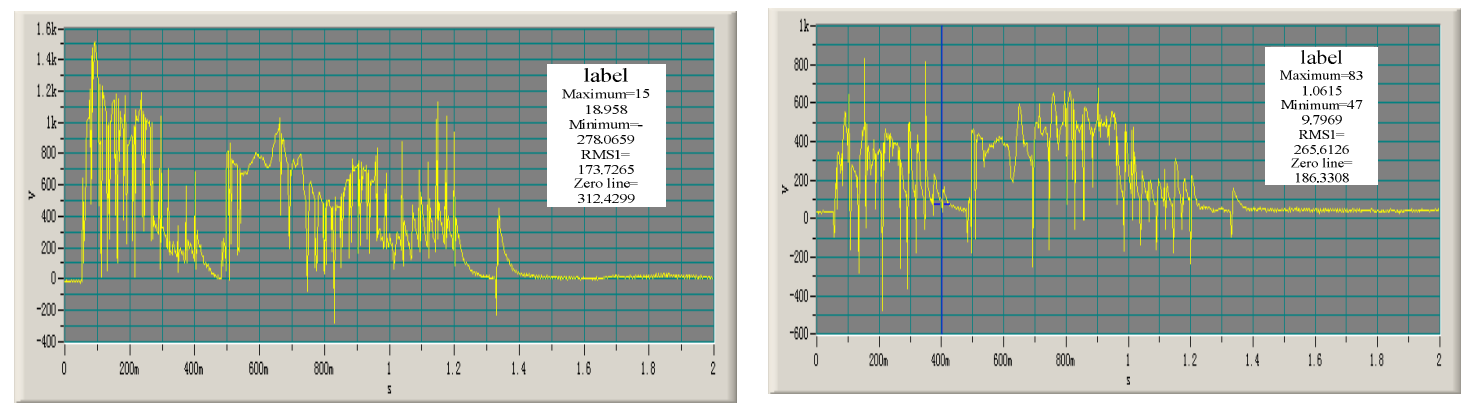

Fig. 9. Measure data curve with heavyload
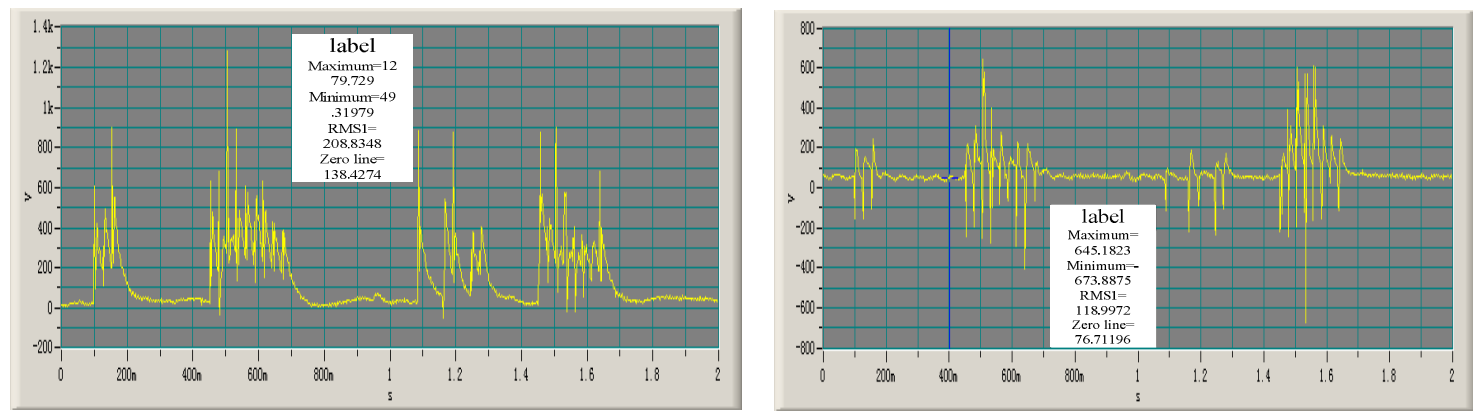

Fig. 10. Measure data curve with unload

This experiment in Anshan Qidashan iron ore factory on the site, R2 new dumper frame of R170 models was selected to test, and start the car, to finish after the strain gauge, stick with the dumper generator, the power supply provided connection data lines. And then, enter into crops unload through the forklift truck operation no-load to be loaded, because seven times, each time load carrying weight unequal, total range of about 190 tons. The test process, from loading no-load start, entruck, transportation, unloading, has been in the dumper to start, the test most is the dynamic testing including the frame dynamic testing of engine and pavement vibration. The test results with different conditions were shown in Fig.8, Fig.9 and Fig.10.

Two of the three conditions are dumper in operation process of the measured data; suffer the mineral impact load, road roughness motivation, engine vibration impact, as a result of the test data to better reflect the stress of the frame. Through the maximum of the three conditions that drive the dumper produce stress less than need stress intensity and won't produce strength damage. In heavy conditions extractive 1, 2 point of the corresponding node stress value were 192.64 Mpa, 193.47 Mpa, the value measured values are in range, explain theory analysis model can reflect the basic frame of the stress state. 
Table 2 Experiment stress parameters

\begin{tabular}{c|c|c|c|c|c|c}
\hline \multirow{2}{*}{ Station } & \multicolumn{2}{|c|}{ noload $(\mathrm{MPa})$} & \multicolumn{2}{c|}{ heavyload $(\mathrm{MPa})$} & \multicolumn{2}{c}{ unload $(\mathrm{MPa})$} \\
\cline { 2 - 7 } & $\max$ & $\min$ & $\max$ & $\min$ & $\max$ & $\min$ \\
\hline 1 & 87.62 & 53.71 & 235.14 & 186.32 & 109.55 & 64.27 \\
\hline 2 & 96.18 & 63.45 & 207.34 & 154.68 & 110.35 & 67.59 \\
\hline
\end{tabular}

Through to the 1, 2 point stress value comparison shows, the two nodes stress level mutual closer, because station decorate up and down, suffer goods impact load and the ground roughness affect order different, bearing strength also is a little different. Such as no-load, frame is mainly affected by the ground not pingdu vibration, 2 nodes in the junction to bottom plate and girder, natural stress intensity larger than 1 nodes. The heavy more influence than unloading to two nodes, mainly is in unloading conditions, mainly concentrated in the load surface frame lift shaft and big fights tile seat, but both through the suspension devices send to the rear axle, small influence on the front of the frame.

\section{Conclusion}

The dumper static's characteristics, modal characteristics of frame and dump on the road of the random vibration were analyzed by the finite element method, and the results show that the frame static intensity and rigidity are agree with the requirements, the frame cracking is not caused by the intensity failure. From initial seven orders natural vibration model analysis, the main deformation of frame is bending and twisting deformation. From the frame displacement, velocity, acceleration response power spectrum, and the frame of the rms stress response on the maximum deformation, the frequency of $21.5 \mathrm{~Hz}$ was obtained, the uneven road surface induced the greatest effect, and the frame of the dynamic stress mainly concentrated in the lift shaft and rear axle joints, is the structure most vulnerable to damage areas. From the frame of the static analysis, modal analysis and random response analysis of the whole, the reasons for cracking the frame is not strong enough for the damage caused, but long-term dynamic impact loads and uneven road surface caused the fatigue cracks.

\section{References}

[1] D. Roller: Computer Aided Design Vol. (5) (1991), p.385.

[2] V.Balamurugan, S.Naray anan: Finite Elements in Analysis and Design Vol.37 (2001), p.713.

[3] S.W. Wu,C. H. Chen, S.W. Wu: Finite Elements in Analysis and Design Vol.36 (2000), p.135.

[4] S. Holmberg, B. Nejabat: Materials\&Design Vol.5 (2004), p.361.

[5] J. El-Sayed, H. Kim: Journal of Materials Processing Technology Vol.1(2004), p.79.

[6] B. Craig, M. Pinfold: Advances in Engineering Software Vol.12 (2001), p.903. 